\title{
Valoración del Estado del Suelo en Zona de Bosque Seco Tropical Mediante Técnicas Analíticas y Cromatogramas
}

\author{
Sonia E. Aguirre*, Nelson V. Piraneque y Carlos J. Díaz \\ Universidad del Magdalena, Santa Marta-Colombia. (e-mail: npiraneque@unimagdalena.edu.co; \\ saguirre@unimagdalena.edu.co; carlosjose.cjdh@gmail.com) \\ ${ }^{*}$ Autor a quien debe ser dirigida la correspondencia
}

Recibido Ene. 21, 2019; Aceptado Mar. 22, 2019; Versión final May. 7, 2019, Publicado Dic. 2019

\begin{abstract}
Resumen
Se analizó y evaluó tres usos de suelo: bosque-B, hortalizas-H y grama-G en una zona de bosque seco tropical mediante técnicas analíticas estándar y por cromatografía circular de Pfeiffer. La evaluación de los cromatogramas se realizó mediante caracterización y puntuación de zonas del patrón, seguido de examen gráfico y analítico en diseño factorial. Posteriormente, estos datos se asociaron a las características físicas y químicas del suelo. Los resultados mostraron diferencias respecto al uso. El suelo $\mathrm{H}$, mostró cromatogramas con menor formación de dientes, colores oscuros y menos integración; el suelo B presentó zonas con relaciones catiónicas que mostraron deficiencia inducida de nutrientes y el suelo G, exhibió colores claros, dientes grandes y mejor integración de zonas, con mayor contenido de carbono. El empleo conjunto de las técnicas evaluadas, proporciona un diagnóstico holístico y confiable para la gestión y sostenibilidad del recurso.
\end{abstract} de patrones

\section{Valuation of Soil State in Dry Tropical Forest Zone by Analytical Techniques and Chromatograms}

\begin{abstract}
Three land uses were analyzed and evaluated: Forest-B, Vegetables- $\mathrm{H}$, and Grass- $\mathrm{G}$ in a tropical dry forest area using standard analytical techniques and Pfeiffer's circular chromatography. The chromatogram pattern evaluation was carried out by characterization and punctuation of pattern zones, followed by graphics and analytical examination in a factorial design. Then, the collected data were associated with physical and chemical soil characteristics. The results showed differences regarding use. Soil $\mathrm{H}$ exhibited chromatograms with less tooth formation, dark colors, and less integrality; soil B presented zones with cationic relationships that showed an induced nutrient deficiency, and soil $\mathrm{G}$ exhibited light colors, large teeth, and better integration of zones, with higher carbon content. The use of the evaluated techniques provides a holistic and reliable diagnosis for management and resource sustainability.
\end{abstract}

Keywords: soil uses; Pfeiffer's circular chromatography; soil quality; soil analysis; pattern evaluations 


\section{INTRODUCCIÓN}

La sobreexplotación de agua y suelo es común en el sector rural y afecta directamente las comunidades y reduce el acceso de alimentos, por lo que es necesario ampliar el conocimiento a el fin de buscar estrategias para la gestión sostenible del recurso. Las funciones del suelo se restringen cuando se alteran sus características. Así lo conforma el informe "Estado mundial del suelo" que plasma diez amenazas a nivel global: erosión, pérdida del carbono orgánico (CO), acidificación, contaminación, anegamiento, compactación, sellamiento superficial, salinización, desequilibrio de nutrientes y merma de la biodiversidad, situación que aumenta con la variabilidad climática y la ampliación de la frontera agropecuaria. Sin embargo, un buen manejo del recurso puede reducir su vulnerabilidad (FAO y GTIS, 2016). La dinámica y compleja interacción del recurso evidencia su capacidad para funcionar y sostener la vida, mantener el agua y aire, sustentar la salud humana y el hábitat, lo que la Sociedad Americana de la Ciencia del Suelo denomina calidad (Bautista et al. 2004). No obstante, no existe criterio holístico que integre, conserve y mantenga la funcionalidad del sistema edáfico, por lo contrario, se evidencia ruptura conceptual entre sus características, producción y sostenibilidad, lo que produce la degradación del recurso (Zambon et al. 2017) que afecta de manera directa el bienestar de las comunidades menos favorecidas.

Los indicadores de calidad del suelo orientan la toma de decisiones respecto al uso del recurso, sin embargo, su estudio es particular por lo que es necesario profundizar en el conocimiento local (García, Ramírez y Sánchez, 2012). A pesar de los adelantos y publicaciones en torno al tema, Latinoamérica afronta dificultades para desarrollar estudios detallados lo que dificulta la gestión del suelo y deja en evidencia la carencia de información de numerosos territorios. Existe diversidad de técnicas analíticas para el estudio de suelos, modificadas según las características de los mismos, muchas diseñadas para condiciones específicas, pero se generalizaron. Un ejemplo es la espectroscópica que asociada a datos de infra rojo y que, con el uso de análisis quimio-métrico, logra estimar parámetros edáficos con exactitud. Desafortunadamente, su acceso no es fácil y muy pocos estados proveen su utilización, lo que dificulta su empleo por parte de pequeños productores.

Así mismo, técnicas descriptivas como la cromatografía de suelos, revelan características de los mismos y son herramienta práctica y efectiva de diagnóstico de calidad (Kokornaczyk et al. 2017). Ehrenfried Pfeiffer (1899-1961) desarrolló el test de cromatografía circular (CCP) para el análisis de la calidad de suelos, compost y cultivos (Pfeiffer, 1984) a través de la formación de patrones y su posterior evaluación. Kokornaczyk et al. (2017), Medina et al. (2018) y Restrepo y Pineiro (2011), sugieren que la CCP es una forma simple, rápida y económica que permite observar detalles de la calidad del suelo especialmente en los contenidos de materia orgánica y así, son instrumentos valiosos que muestra elementos que los análisis convencionales no consideran ni describen. Medina et al. (2018) reconocen que está técnica incluye la actividad biológica (microorganismos en procesos bioquímicos) y transformación de elementos minerales que otras técnicas no evidencian y que deben ser estudiada con mayor profundidad.

Restrepo y Pineiro (2011) describieron cuatro zonas de una CCP: Zona 1: central, de oxigenación y estructura, refleja los microorganismos del suelo encargados de descomponer la $\mathrm{MO}$ en humus, si el suelo esta compactado se restringe la circulación de oxígeno y se disminuye la actividad microbiológica, por lo que no existe zona central. El color ideal es blanco crema y es un indicador de adecuada porosidad y estructura. Los cromas oscuros son suelos compactados y no reflejan buenos síntomas, suelen ser excesivamente mineralizados sin cobertura vegetal. Zona 2: Mineral, color crema suave (amarillo), evidencia integración con las otras zonas, pero colores diferentes (oscuros) presentan degradación del suelo, alta mineralización con estructura poco estable y baja actividad biológica. Cuando la microbiología de la zona 1 está interactuando con los minerales zona 2, se observa armonía en color e integración cromática.

Zona 3: Intermedia, proteica o de materia orgánica (M.O), destaca la presencia o la ausencia de la M.O en el suelo. Colores suaves es indicador de armonía y de la presencia de una buena zona enzimática (zona 4) por lo que se facilita la formación de dientes y nubes. Por el contrario, puede tener buena cantidad de M.O. pero esta no estar activa, porque no se dispone de descomponedores para que la transformen y suelos mineralizados; en este caso no hay integración y se presentara tonos oscuros muy distintos entre ellos. Colores oscuros con puntas agudas en lugar de dientes, no son deseables y Zona 4: Externa, enzimática 0 nutricional. Se manifiesta de forma gradual y armónica, con dientes y nubecillas o lunares tenues que indican abundancia y variedad de nutrientes, si se observan tonos café y nubecillas al final de los dientes es la expresión de diversidad microbiológica que permite la formación enzimática y descomposición de MO. Los dientes deben llegar hasta el borde del croma, acompañados de nubecillas. Si las nubecillas están separadas de los dientes, indican abundante comida en el suelo de forma estable por mucho tiempo, lo cual es una característica muy deseada. 
Por otro lado, la complementariedad de técnicas cualitativas y cuantitativas se entrelaza en diferentes disciplinas, una de ellas es la estadística que categoriza y codifica datos con el fin de explicar fenómenos e interpretar significados (González y Ruiz, 2011). Bajo este criterio se puede mejorar la conceptualización de los ecosistemas, ya que no es suficiente caracterizar parámetros, sino que se necesita una base robusta de información y un análisis sistémico para profundizar en la interpretación y complejidad de los recursos (Smith et al. 2016). En Colombia, la región Caribe es una de las zonas más vulnerables a la degradación edáfica debido a su geomorfología, posición geográfica con cercanía al mar, temperatura alta, desforestación y el inadecuado manejo del recurso han deteriorado su territorio. Al respecto, el Instituto de Hidrología, Meteorología y Estudios Ambientales (IDEAM y U.D.C.A, 2015), advierte que el departamento del Magdalena presenta índices de erosión del 76,9\% con alta susceptibilidad a salinización y desertificación.

Dado el papel del suelo en la regulación hídrica y de ciclos biogeoquímicos, en el soporte para la vegetación y como sumidero de Carbono (Rossel et al. 2010; Keesstra et al. 2016; Jia et al. 2017), es necesario caracterizar y monitorear el recurso. Al respecto, Vásquez y Macías (2017) detectaron pérdidas de Carbono total edáfico en el área de estudio de un $26 \%$, en otras palabras, el suelo se está degradando. Por lo anterior, el objetivo de este trabajo fue evaluar la calidad del suelo en una zona de bosque seco tropical con tres sistemas de uso diferentes: bosque $(B)$, hortícola $(H)$ y gramas $(G)$, mediante técnicas analíticas estándar (TAS) y el uso de CCP.

\section{METODOLOGÍA}

Se describe la ubicación de la investigación, las técnicas de muestreo el procesamiento realizado para determinar la calidad del suelo según uso y se explica finalmente, el análisis estadístico realizado.

\section{Área de estudio}

El trabajo se realizó durante el año 2017 en el campus de la Universidad del Magdalena - Santa Marta, Colombia (Figura 1), zona de vida del Bosque Seco Tropical con régimen de precipitación bimodal y precipitación media mensual de $578 \mathrm{~mm}$, temperatura promedio anual de 29두 ; clima semi-árido con marcado déficit hídrico en la época seca (Rangel y Carvajal, 2012). El campus cuenta con 60 ha, de éstas 10 están ocupadas por edificaciones entrelazadas por gramas y un lago artificial, 17 pertenecen al Centro de Servicios Agropecuarios con diferentes cultivos, entre ellos hortalizas y 3 ha están dedicadas a reserva natural denominada bosque. Los suelos de la zona son de origen aluvial, poco evolucionados con predominio de texturas gruesas, (franco arcillo arenosa), clasificados en el orden Entisoles y suborden Psamments; Vásquez, Baena y Menjivar (2010) los clasificaron como Typic Ustipsamments. Las zonas de estudio fueron: natural, que corresponde a relictos de bosque seco tropical reserva ecológica del campus denominado bosque (B) con más de 20 años de edad. Sistemas agrícolas, que corresponden a lotes de hortalizas $(H)$ con un manejo intensivo convencional en riego, control fitosanitario, fertilización y preparación del terreno usados por más de 25 años; y un tercer uso, suelos de zonas verdes o gramas $(G)$ empleadas para el esparcimiento establecidas hace aproximadamente 15 años.

\section{Muestreo}

Se realizó muestreo aleatorio estratificado, el área de estudio se subdividió en tres sitios y usos principales conformando los estratos o subgrupos homogéneos en el terreno y en cada uno de ellos se efectúo un muestreo aleatorio simple en cinco puntos o replicas. Cada punto fue georreferenciado, se realizó cajuela de $40 \mathrm{~cm}$ (largo) x $40 \mathrm{~cm}$ (ancho) $\times 40 \mathrm{~cm}$ (profundidad) de donde se recolectaron muestras disturbadas y sin disturbar a dos profundidades $(0$ a $10 \mathrm{~cm}$ y de 10 a $30 \mathrm{~cm}$ ), para un total de 90 muestras.

\section{Medición de parámetros químicos y físicos}

En campo, con multiparámetro, se determinó conductividad eléctrica, temperatura y humedad, mientras que en laboratorio de suelos de la Universidad del Magdalena se determinó densidad aparente, pH (relación sueloagua 1:2,5), Carbono orgánico (CO) por el método de digestión en húmedo (Walkley y Black, 1934). Adicionalmente, en el Centro Internacional de Agricultura Tropical (CIAT), se determinó nitrógeno total (Nt) por combustión, fosforo disponible (P) por Bray II espectrometría. Cationes Intercambiables calcio, magnesio, sodio y potasio ( $\mathrm{Ca}, \mathrm{Mg}$, Na y $\mathrm{K}$, respectivamente): extracción con acetato de amonio, $1 \mathrm{~N}, \mathrm{pH} 7$ por espectroscopia de absorción atómica y los elementos menores hierro, cobre, manganeso y cinc ( $\mathrm{Fe}, \mathrm{Cu}, \mathrm{Mn}$ y Zn, respectivamente): extracción Mehlich (espectroscopia de absorción atómica). 


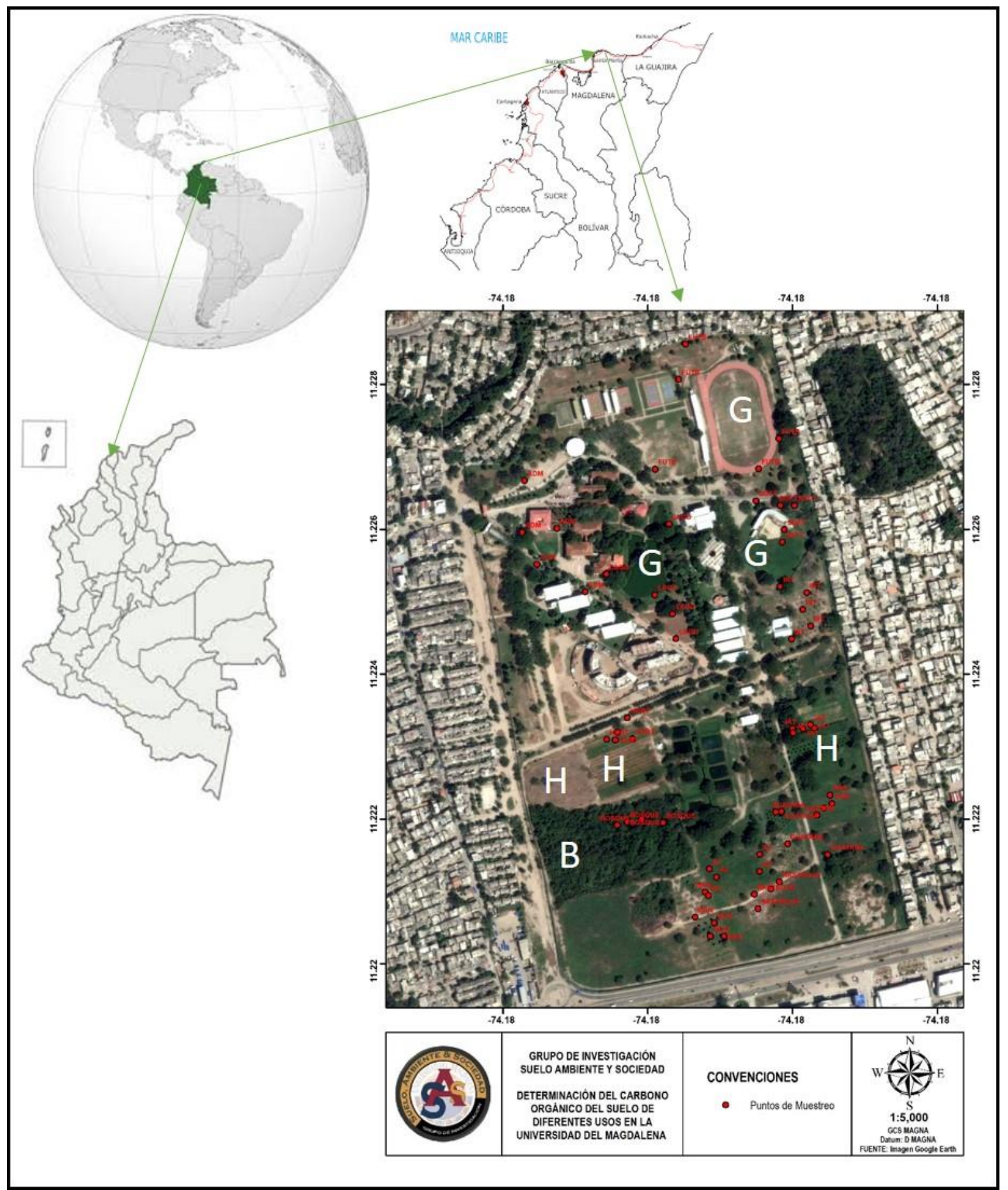

Fig. 1. Localización, zonas y usos del suelo en el campus Universidad del Magdalena (Adaptado de Google earth, 2018)

\section{Test de Cromatografía Circular de Pfeiffer}

En el laboratorio de suelos de la Universidad del Magdalena se realizaron los CCP en papel filtro Whatman de $150 \mathrm{~mm}$ de diámetro, de acuerdo a la metodología descrita por Restrepo y Pineiro (2011) y Kokornaczyc et al. (2017) y se caracterizaron las muestras por sistema. Para esto, se observó la formación de diferentes zonas o círculos radiantes (Figura 2) y se procedió a realizar la descripción; color, formación de dientes e integración de las zonas de los CCP, por lo que fue pertinente tener en cuenta particularidades por cada zona. En la parte superior de la figura 2 se muestra las Zonas de CCP de suelo de Santa Marta: 1: Zona central de oxigenación o de estructura del suelo; 2: Zona se interna o zona mineral; 3: Zona se llama intermedia, proteica o zona de materia orgánica; y 4: Zona externa, enzimática o nutricional. En la parte inferior de la figura 2 se presentan las características ideales de un CCP: A) Integración y armonía en todas las zonas; B) Dirección integrada de caminos forma de plumas; C) Manchas entre dientes, disponibilidad de nutrientes; y D) Diversidad de tamaño y diversidad de dientes. 


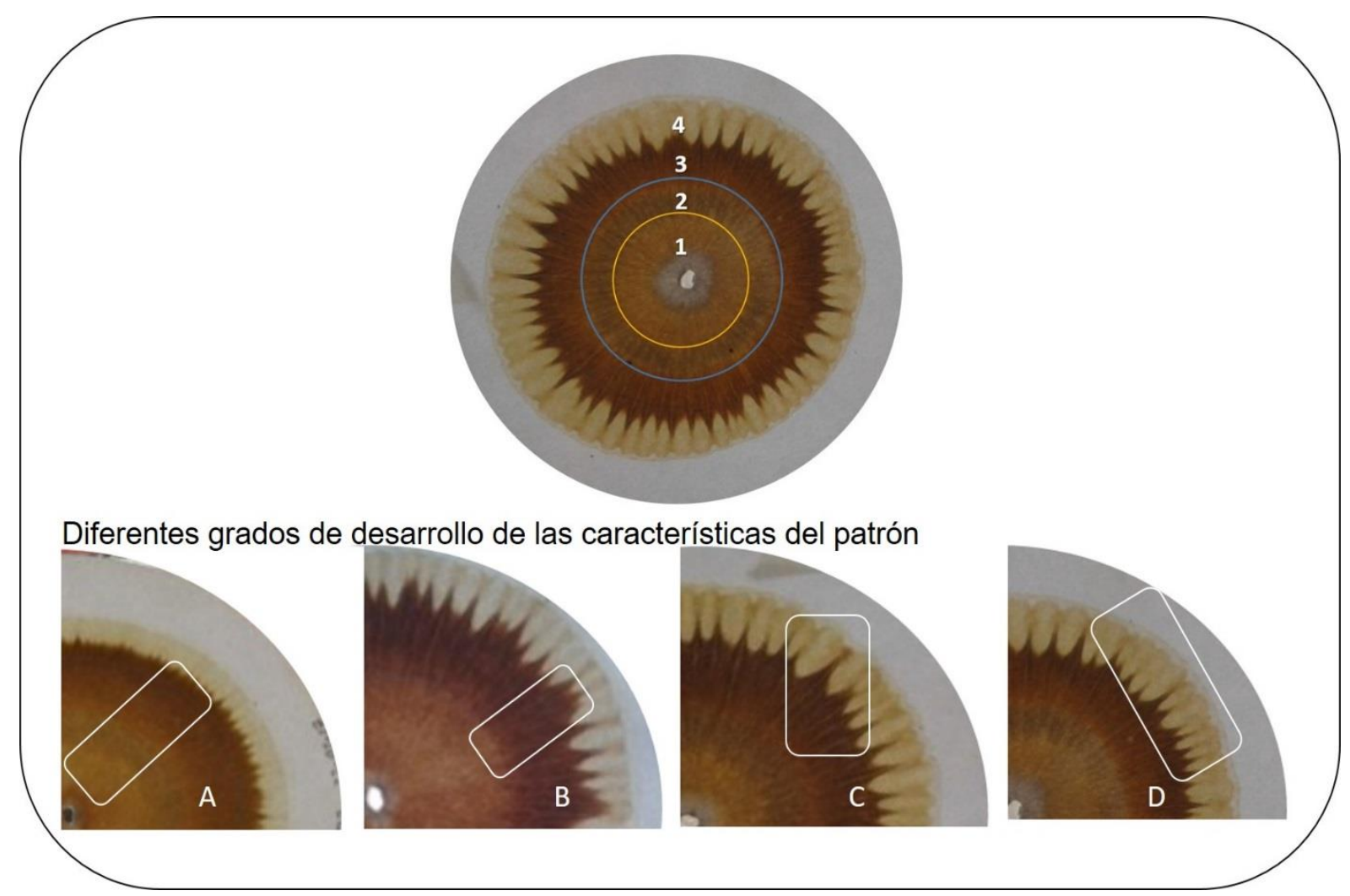

Fig. 2. Zonas y características del CCP empleadas para el análisis cualitativo y diagnóstico del suelo en diferentes sistemas de producción en área de bosque seco tropical de Santa Marta. Adaptado de Restrepo y Pinheiro (2011) y Kokornaczyk et al. (2017).

En la parte superior de la figura 2 se muestra las Zonas de CCP de suelo de Santa Marta: 1: Zona central de oxigenación o de estructura del suelo; 2: Zona se interna o zona mineral; 3: Zona se llama intermedia, proteica o zona de materia orgánica; y 4: Zona externa, enzimática o nutricional. En la parte inferior de la figura 2 se presentan las características ideales de un CCP: A) Integración y armonía en todas las zonas; B) Dirección integrada de caminos forma de plumas; C) Manchas entre dientes, disponibilidad de nutrientes; y D) Diversidad de tamaño y diversidad de dientes.

\section{Análisis estadístico de la información}

Para valorar el recurso a partir de los CCP, se planteó una escala de evaluación (Tabla 1) para lo que se otorgó valores a las características de color, presencia de dientes, nubecillas e integración de las zonas. Para establecer la relación entre las variables medidas con el uso del suelo, se acudió a interpretación gráfica y análisis de varianza $(p<0,05)$ de cada variable medida seguida de una prueba post hoc de Tukey $(p<0,05)$. Con el fin de explicar las relaciones entre las variables observadas en términos de un número menor de variables, se empleó análisis multivariado con diseño factorial donde los factores fueron los usos del suelo ( $B$, $H$ y G) y las dos profundidades estudiadas $(0-10 \mathrm{~cm}$ y $10-30 \mathrm{~cm}$ ) seguida de análisis clúster a fin de clasificar las variables según el uso, se empleó distancia euclidea como indicador de disimilaridad. Los análisis se realizaron con el programa estadístico R versión 3.02 ( $R$ Development Core Team 2015 disponible en: www.rproject.org) y la versión libre de Infostat 2017.

Tabla 1. Escala de evaluación de CCP utilizado para los diferentes sistemas de producción en un Typic ustipsamments de bosque tropical seco.

\begin{tabular}{|l|l|l|l|l|l|}
\hline Característica 1 & Característica 2 & \multicolumn{2}{l|}{ Característica 3 } \\
\hline Color (C) & Grado & Tipo de Dientes (D) & Grado & Diferenciación de Zonas (Z) & Grado \\
\hline $\begin{array}{l}\text { Amarillo-Claro } \\
\text { resplandeciente }\end{array}$ & 3 & $\begin{array}{l}\text { Dientes en forma de diente } \\
\text { de caballo }\end{array}$ & 3 & $\begin{array}{l}\text { Presenta 4 zonas bien } \\
\text { definidas }\end{array}$ & 3 \\
\hline Amarillos opacos & 2 & Dientes en forma de maíz & 2 & Presenta 3 zonas & 2 \\
\hline Oscuros & 1 & $\begin{array}{l}\text { Dientes en Aguja angular } \\
\text { puntuda }\end{array}$ & 1 & Presenta 2 zonas & 1 \\
\hline $\begin{array}{l}\text { Colores opacos } \\
\text { marrones }\end{array}$ & 0 & Plano sin bordes-circular & 0 & No se distinguen zonas & 0 \\
\hline
\end{tabular}




\section{RESULTADOS Y DISCUSIÓN}

En la Tabla 2 se muestran los resultados que se obtuvieron a partir de las TAS. En ella se presentan los valores promedio de las muestras de suelo analizadas a dos profundidades $(0-10 \mathrm{~cm}$ y $10-30 \mathrm{~cm})$ para cada sistema de producción.

Tabla 2. Parámetros físicos y químicos de los suelos evaluados por métodos analíticos convencionales en un Typic ustipsamments de bosque tropical seco.

\begin{tabular}{|l|l|l|l|l|l|l|l|}
\hline Parámetro & \multicolumn{9}{|c|}{ Sistema } \\
\hline & Unidad & \multicolumn{2}{|l|}{ Bosque B } & \multicolumn{2}{l|}{ Gortalizas $\mathrm{H}$} & \multicolumn{2}{l|}{ Gramas G } \\
\hline Profundidad & $\mathrm{cm}$ & $0-10$ & $10-30$ & $0-10$ & $10-30$ & $0-10$ & $10-30$ \\
\hline $\mathrm{pH}$ & & 7.52 & 7.84 & 8.14 & 7.86 & 7.75 & 7.96 \\
\hline Humedad & $\%$ & 13 & 17.5 & 14.4 & 13.96 & 12.7 & 12.5 \\
\hline $\mathrm{Da}$ & $\mathrm{g} / \mathrm{cm}^{-3}$ & 1.35 & 1.46 & 1.66 & 1.76 & 1.58 & 1.62 \\
\hline $\mathrm{CE}$ & $\mathrm{dS} \mathrm{m}^{-1}$ & 0.41 & 0.37 & 0.52 & 0.38 & 0.53 & 0.41 \\
\hline $\mathrm{ClC}$ & $\mathrm{cmol}^{-}+\mathrm{kg}^{-1}$ & 33.0 & 26.4 & 18.8 & 17.2 & 36.0 & 24.4 \\
\hline $\mathrm{CaC}{ }_{3}$ & $\%$ & 22.0 & 17.0 & 22.0 & 19.0 & 20.0 & 18.0 \\
\hline $\mathrm{Nt}$ & $\%$ & 0.18 & 0.14 & 0.15 & 0.19 & 0.18 & 0.16 \\
\hline $\mathrm{CTS}$ & $\%$ & 1.22 & 0.87 & 1.19 & 1.09 & 1.52 & 1.38 \\
\hline $\mathrm{P}$ & $\mathrm{mg} \mathrm{kg}^{-1}$ & 0.90 & 1.20 & 5.03 & 8.52 & 12.5 & 14.0 \\
\hline $\mathrm{K}$ & $\mathrm{mg} \mathrm{kg}^{-1}$ & 5.00 & 9.00 & 51.0 & 37.0 & 58.0 & 54.0 \\
\hline $\mathrm{Ca}$ & $\mathrm{mg} \mathrm{kg}^{-1}$ & 160 & 176 & 158 & 135 & 211 & 181 \\
\hline $\mathrm{Mg}$ & $\mathrm{mg} \mathrm{kg}^{-1}$ & 22.3 & 35.0 & 21.0 & 18.0 & 12.4 & 11.4 \\
\hline $\mathrm{Na}$ & $\mathrm{mg} \mathrm{kg}^{-1}$ & 3.66 & 4.76 & 15.78 & 16.1 & 10.2 & 11.2 \\
\hline $\mathrm{S}$ & $\mathrm{mg} \mathrm{kg}^{-1}$ & 13.3 & 8.14 & 29.79 & 36.3 & 17.4 & 12.5 \\
\hline $\mathrm{Fe}$ & $\mathrm{mg} \mathrm{kg}^{-1}$ & 0.04 & 0.09 & 0.088 & 0.07 & 0.09 & 0.08 \\
\hline $\mathrm{Zn}$ & $\mathrm{mg} \mathrm{kg}^{-1}$ & 0.09 & 0.07 & 0.043 & 0.01 & 0.04 & 0.05 \\
\hline $\mathrm{Cu}$ & $\mathrm{mg} \mathrm{kg}^{-1}$ & 0.02 & 0.01 & 0.005 & 0.21 & 0.03 & 0.01 \\
\hline $\mathrm{Mn}$ & $\mathrm{mg} \mathrm{kg}^{-1}$ & 0.09 & 0.57 & 0.922 & 1.09 & 0.19 & 0.19 \\
\hline $\mathrm{B}$ & $\mathrm{mg} \mathrm{kg}^{-1}$ & 0.05 & 0.04 & 0.070 & 0.07 & 0.09 & 0.07 \\
\hline
\end{tabular}

Una característica importante del suelo es la densidad toda vez que se relaciona de manera directa con retención de humedad, intercambio de gases, transporte y asimilación de nutrientes. Esta variable reportó diferencias entre los sistemas evaluados, en bosque (B) se alcanzaron los valores más bajos con $1.355 \mathrm{~g} \mathrm{~cm}^{-}$ ${ }^{3}$ seguido de $1.578 \mathrm{~g} \mathrm{~cm}^{-3}$ en gramas $(G)$ y $1.66 \mathrm{~g} \mathrm{~cm}^{-3}$ en hortalizas $(\mathrm{H})$. Se infiere que incrementos en los valores de la variable pueden explicarse por la intervención (mecanización) del recurso; al respecto, Wolf y Snyder (2003) afirman que prácticas de labranza en textura arcillosa, relacionan de manera directa con densidad, así mismo, Nikolaidis (2011), expone como los sistemas productivos tradicionales, degradan las propiedades físicas e hidrodinámicas del recurso, así como las funciones eco-sistémicas.

La densidad encontrada en $\mathrm{H}\left(1.66 \mathrm{~g} \mathrm{~cm}^{-3}\right)$ refleja disminución del espacio poroso lo que influye de manera negativa en la circulación de agua y aire y, por tanto, en la función hidrológica del sistema lo que coincide con lo reportado por Medina et al. (2018) quienes afirman que este tipo de suelos se encuentran afectados por la intensificación de la producción, la profundidad de la mecanización y el amplio uso de fertilizantes y defensores químicos. Respecto al $\mathrm{pH}$, los sistemas evaluados presentaron diferencias significativas. $\mathrm{G}$ se diferenció de $\mathrm{H}$ y B, sin embargo, todos superan la neutralidad lo que concuerda con las características de los materiales de formación (calcáreos) que se acentúan por las condiciones climáticas (semiáridas) del área de estudio en donde abundan bases. En pH mayores a 7.5 se insolubiliza el hierro Briat, Dubos y Gaymard, 2015) y se restringe la nutrición vegetal dado que la disponibilidad de $\mathrm{P}, \mathrm{Mg}$ y $\mathrm{Ca}$ no es eficiente y existe riesgo de formación de sales y/o carbonatos, además se disminuye de la actividad microbiológica y la nutrición de las plantas se ve seriamente afectada. 
El contenido de Nt no mostró diferencias estadísticas, sus concentraciones son muy bajas y disminuye con la profundad, lo cual está relacionado con bajos contenidos de CO del suelo y escasa actividad biológica. Valores superiores a $0.22 \%$ de Nt para ecosistemas áridos son consideras altos y por debajo de $0.032 \%$ de Nt muy pobres (Garratt et al. 2018), en este sentido, los suelos son pobres en MO que, unido a las temperaturas altas y la escasa precipitación, incrementa su mineralización y afecta la producción de biomasa (cobertura vegetal) e incrementa la degradación (Zambón et al. 2017). El Carbono Total del Suelo CTS es pobre en los tres sistemas evaluados, se observó que G $(1.52 \%)$ superó a B y H. Lo anterior coincide con Nagargade, Tyagi y Kumar (2017) quienes argumentan que el empleo de sistemas de cobertura vegetal permanente, es una de las estrategias para elevar la concentración de CO en el suelo y así, la captura de $\mathrm{CO}_{2}$ atmosférico. Por otra parte, el contenido de $\mathrm{MO}$ es indicador de calidad, está directamente relacionado con las funciones ecológicas del suelo. A mayor contenido de MO aumenta el "carbono vivo" y la actividad biológica lo que incide en la estructura, estabilidad de los agregados, capacidad de regulación térmica, suministro de agua y nutrientes a las plantas (Jia et al. 2017; Garratt et al. 2018). Dado el bajo contenido en MO del suelo en estos sistemas se puede inferir que la fracción arcilla es la responsable de la capacidad de cambio. Precisamente, en G se presentó el mayor valor de $\mathrm{CIC}\left(36 \mathrm{cmol}_{+} \mathrm{kg}^{-1}\right)$ posiblemente por el aporte de material orgánico (ciclaje de raíces y hojas) que genera la cobertura permanente del suelo.

Los contenidos de $\mathrm{P}$ evidenciaron diferencias significativas entre sistemas siendo $\mathrm{G}$ con $13,25 \mathrm{mg} \mathrm{kg}^{-1}$ el que mostró mayor concentración. Costa et al. (2014) afirman que este elemento es poco móvil en el suelo y no es insólito encontrarlo en concentraciones más altas en superficie, sobre todo en aquellos sistemas destinados a labores agrícolas donde se fertiliza como lo reportó H y G que mostraron los más altos valores. Sin embargo, contrario a lo descrito, los contenidos encontrados son bajos a medios y aumentan en profundidad. Los resultados evidenciaron que los micronutrientes presentaron secuencia de los niveles litológicos y su concentración se encuentra en rango normal (Tabla 2), lo que es de vital importancia dada su relación con la fisiología y la respuesta bioquímica de las plantas (Sas et al. 2003).

El análisis de las relaciones de cationes $\mathrm{Ca}, \mathrm{Mg}$ y $\mathrm{K}$ a nivel edáfico es importante en nutrición vegetal, Bertsch (1987) y Molina (2007) plantean antagonismo y sinergismo entre estos elementos por lo que consideran algunas categorías en los que se han encontrado las mejores condiciones para su absorción. En la Tabla 3 se muestran los niveles de referencia con que fueron comparados los encontrados en los diferentes sistemas. Al respecto, cuando la relación $\mathrm{Ca} / \mathrm{Mg}$ es mayor a 4, implica deficiencia inducida de Magnesio. Asimismo, si la relación $\mathrm{Mg} / \mathrm{K}$ es menor a 1 (como en los sistemas $\mathrm{H}$ y G), evidencia deficiencia de Mg (Mengel y Kirkby, 2006). Cuando la relación $\mathrm{Ca} / \mathrm{K}$ es mayor a 30 (como en el sistema $\mathrm{B}$ ), se genera deficiencia de $\mathrm{K}$. De acuerdo a lo descrito, se pueden presentar deficiencias inducidas de $\mathrm{Mg}$ y $\mathrm{K}$ por efectos antagónicos dada la alta concentración de $\mathrm{Ca}$ en el suelo afectando notoriamente la producción vegetal del área en estudio. El Na, no es nutriente esencial, algunas especies muestran respuesta positiva y se argumenta que puede suplantar al $\mathrm{K}$ en ciertas funciones, pero cuando hay exceso, los coloides se dispersan se desagrega y aumenta la concentración de sal $(\mathrm{NaCl})$, que compite con el potasio, el calcio, el magnesio y el amonio por sitios específicos de absorción, un contenido elevado en la solución del suelo, no es conveniente. Al respecto, se evidenció diferencia significativa entre sistemas y profundidad, siendo $\mathrm{H}$ con $15.78 \mathrm{mg} \mathrm{kg}^{-1} \mathrm{y} 16.10 \mathrm{mg} \mathrm{kg}^{-1} \mathrm{el}^{-1}$ que mostró mayor valor en la variable en las profundidades 0-10 y 10-30 cm, respectivamente.

Tabla 3. Relaciones $\mathrm{Ca}, \mathrm{Mg}$ y $\mathrm{K}$ en los sistemas de producción de bosque, hortalizas y gramas encontrados en suelos de bosque tropical seco comparados con los rangos ideales reportados por Bertsch, F. (1987) y Molina, E. (2007).

\begin{tabular}{|c|c|c|c|c|}
\hline \multicolumn{5}{|c|}{ Relaciones } \\
\hline Rangos ldeales & $\mathrm{Ca} / \mathrm{Mg}$ & $\mathrm{Mg} / \mathrm{K}$ & $\mathrm{Ca} / \mathrm{K}$ & $\mathrm{Ca}+\mathrm{Mg} / \mathrm{K}$ \\
\cline { 2 - 5 } & $2 \mathrm{a} \mathrm{4}$ & $3 \mathrm{a} 15$ & $5 \mathrm{a} \mathrm{25}$ & $10 \mathrm{a} 40$ \\
\hline Bosque & 7.1 & 4.46 & 32.0 & 36.5 \\
\hline Hortalizas & 7.5 & 0.41 & 3.09 & 3.50 \\
\hline Gramas & 17 & 0.21 & 3.63 & 3.85 \\
\hline
\end{tabular}

Por lo anterior, se determinó la concentración de carbonatos, los cuales se encontraron cercanos a $20 \%$. Estos tienen acción positiva en estructura y en la actividad de microorganismos, pero cuando hay excesos acarrean deficiencia de elementos. En pH que oscila entre 7.5 y 8 es común encontrar carbonato de Ca, mientras que a $\mathrm{pH}$ superiores a 8.5 predomina el carbonato sódico. El porcentaje de carbonatos normal oscila de 10 a 20; un porcentaje cercano a 20, se considera alto (Andrades y Martínez, 2001). Así las cosas, la concentración de $\mathrm{CaCO}_{3}$ encontrado en estos suelos (alrededor de $20 \%$ ), constituye un factor de riesgo. A pesar de no haber diferencia estadística para este parámetro entre los sistemas de usos evaluados, los contenidos altos, unido a las condiciones intrínsecas del ecosistema, incrementa la vulnerabilidad del territorio. Finalmente, los valores de conductividad eléctrica (CE) medidos son bajos y se encuentran dentro de la normalidad $\left(<4 \mathrm{dS} \mathrm{m}^{-1}\right)$, sin embargo, se presentó diferencias entre los sistemas donde $\mathrm{H}$ y $\mathrm{G}$ con mayor valor en la variable, se diferenciaron de $\mathrm{B}$ con los menores promedios. 
El análisis determinó que tres factores explicaron el $85 \%$ de la variabilidad y que las variables que definen al grupo $\mathrm{G}$ (grama) están vinculadas a la relación $\mathrm{Ca} / \mathrm{Mg}$, al contenido de $\mathrm{P}, \mathrm{CTS}$ y $\mathrm{K}$. Mientras que el grupo $\mathrm{B}$ (Bosque) se encuentra influenciado por contenido de $\mathrm{Mg}$, $\mathrm{Ca}$ y sus relaciones catiónicas y el grupo H (Hortalizas), está relacionado con la concentración de Na y densidad (Figura 3).
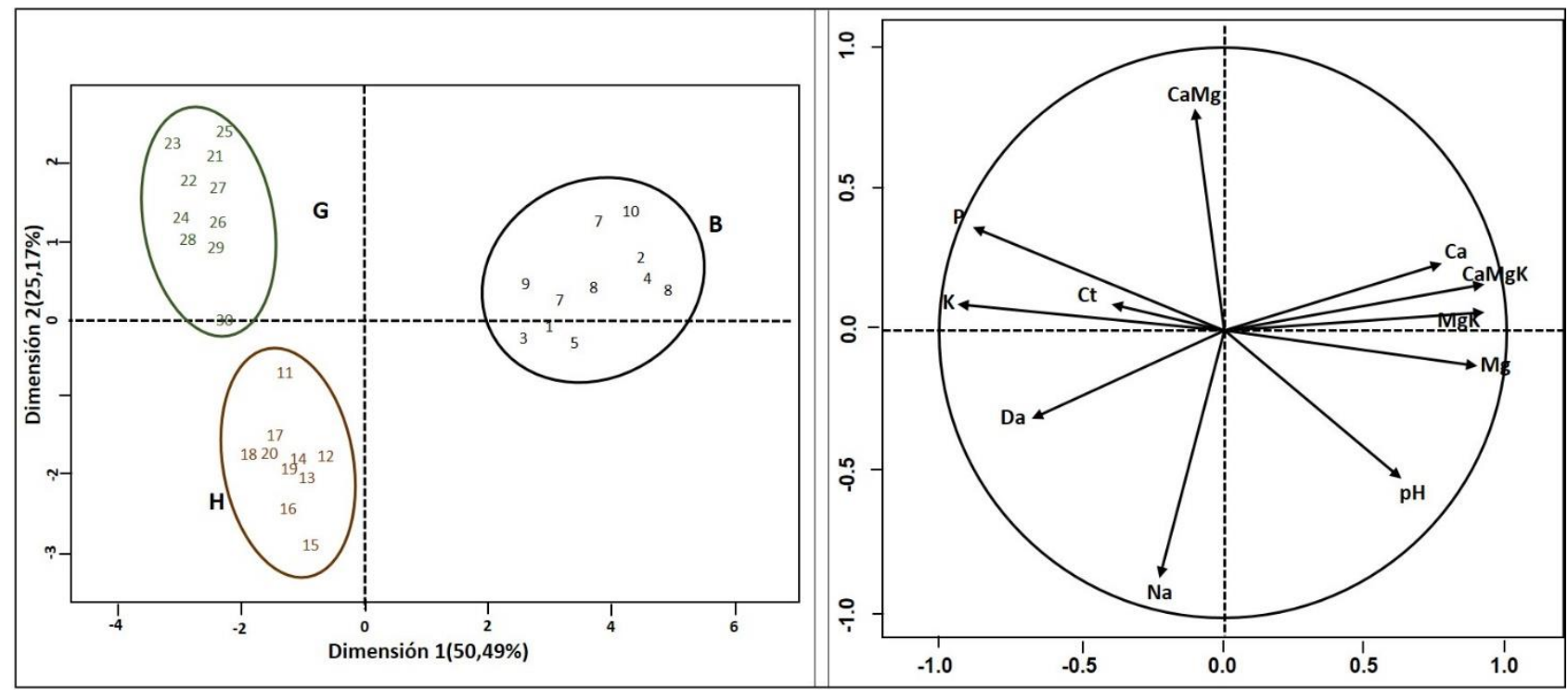

Fig. 3. Derecha: Ordenamiento de los sistemas de producción bosque (B), hortalizas $(H)$ y gramas (G) basado en las variables cuantitativas medidas. Izquierda: proyección vectorial de las variables medidas y su relación con los sistemas evaluados.

En la Figura 4 se aprecian algunos CCP analizados y caracterizados en los diferentes sistemas y que luego fueron valorados de acuerdo a los rangos establecidos en la tabla 1. De forma general se observó colores amarillos opacos y marrones, dientes en forma de maíz y angulares y de 4 a 3 zonas que en ocasiones se integran o por el contrario presenta una forma marcada respecto a las demás, lo que denota desintegración con bajo porcentaje de MO. La última zona (4) presento dientes de color café claro a muy oscuros, pero rara vez son acompañados de nubecillas por lo que se infiere baja actividad y diversidad microbiológica de los sistemas analizados.

En la Figura 5 se observan valores promedios de las características evaluadas (color, dientes y diferenciación por zonas) por cada sistema a dos profundidades. El sistema $\mathrm{G}$ mostró mayores promedios en las variables color, dientes e integración de las zonas evidenciando mejor comportamiento del sistema. Muestra que a medida que las características evaluadas (color, dientes y zonas en los CCP) se agrupan, el funcionamiento del sistema es más estable, lo que significa que las características son menos disímiles lo que representa más interacción, en otras palabras, mejor comportamiento sistémico del recurso o, al igual que reporta Kornaczyk et al. (2017), aquellos CCP con características radiales se relacionan con características positivas en el suelo como altos contenidos de materia orgánica (como se presentó en G), mientras que en aquellos CCP donde existe patrón concéntrico marcado, indican pobre calidad del suelo (como lo mostró H).

De acuerdo a lo encontrado, el sistema $\mathrm{H}$ (hortalizas) reportó valores más altos de densidad aparente y sodio, además, presentó valores desiguales en los promedios respecto a las características evaluadas por cromatografía, con valores altos en Z (zonas) respecto a color y dientes, lo que pone de manifiesto la falta armonía, posiblemente la diversidad biológica está restringida y la formación enzimática es escasa por lo que el comportamiento del recurso no es sistémico, lo que se confirma con los análisis estadísticos donde se evidenció la conformación de dos grupos (Figura 6), hortalizas y grama-bosque, con comportamiento diferente para las variables evaluadas, conformando dos conglomerados donde el análisis permitió apreciar una relación estrecha entre color y dientes en los sistemas evaluados, mostrando con los menores ángulos de los vectores color y dientes (gráfico parte inferior derecha), e indica que presentan características similares y que son éstas las que diferencian los sistemas de producción. Así, $\mathrm{H}$ evidenció menor promedio en color y en dientes, respecto a $\mathrm{B}$ y G. De lo anterior se infiere, que la integración y el número de zonas en los CCP son factor primordial que establece la unidad y comportamiento del sistema y de esta manera, esta cualidad marcó la diferencia en los sistemas analizados. 


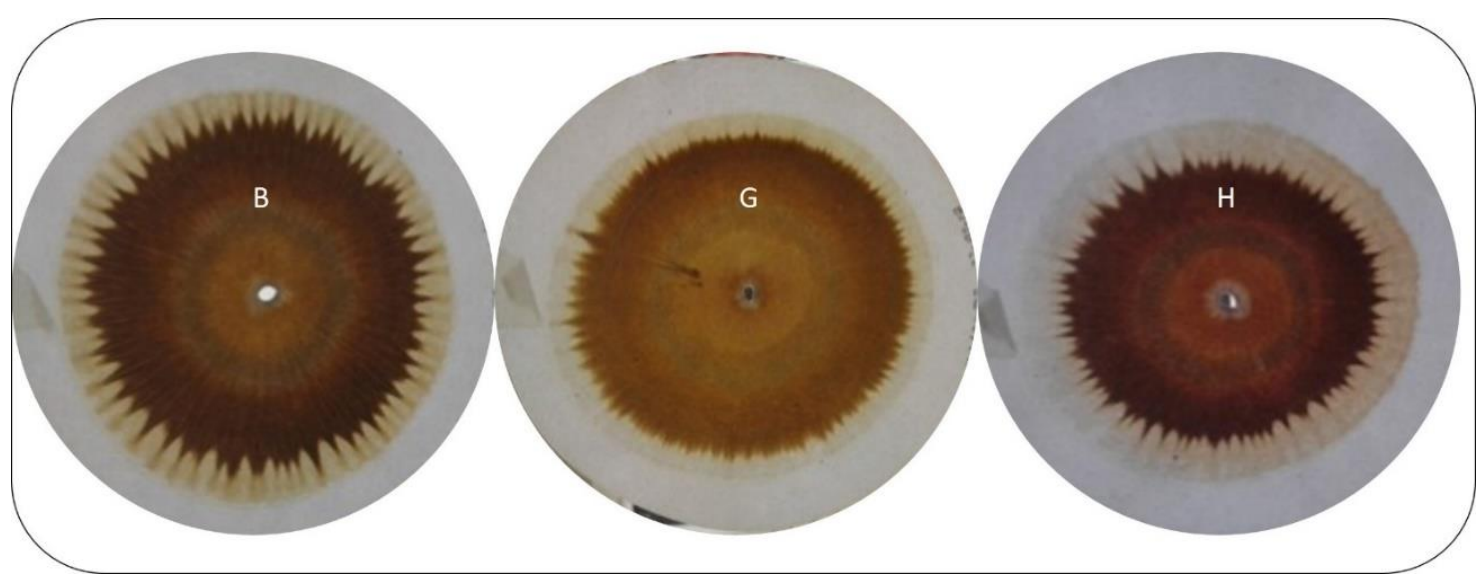

Fig. 4. CCP de los sistemas B, G y H, evaluados en una zona de bosque seco tropical de Santa Marta - Colombia.

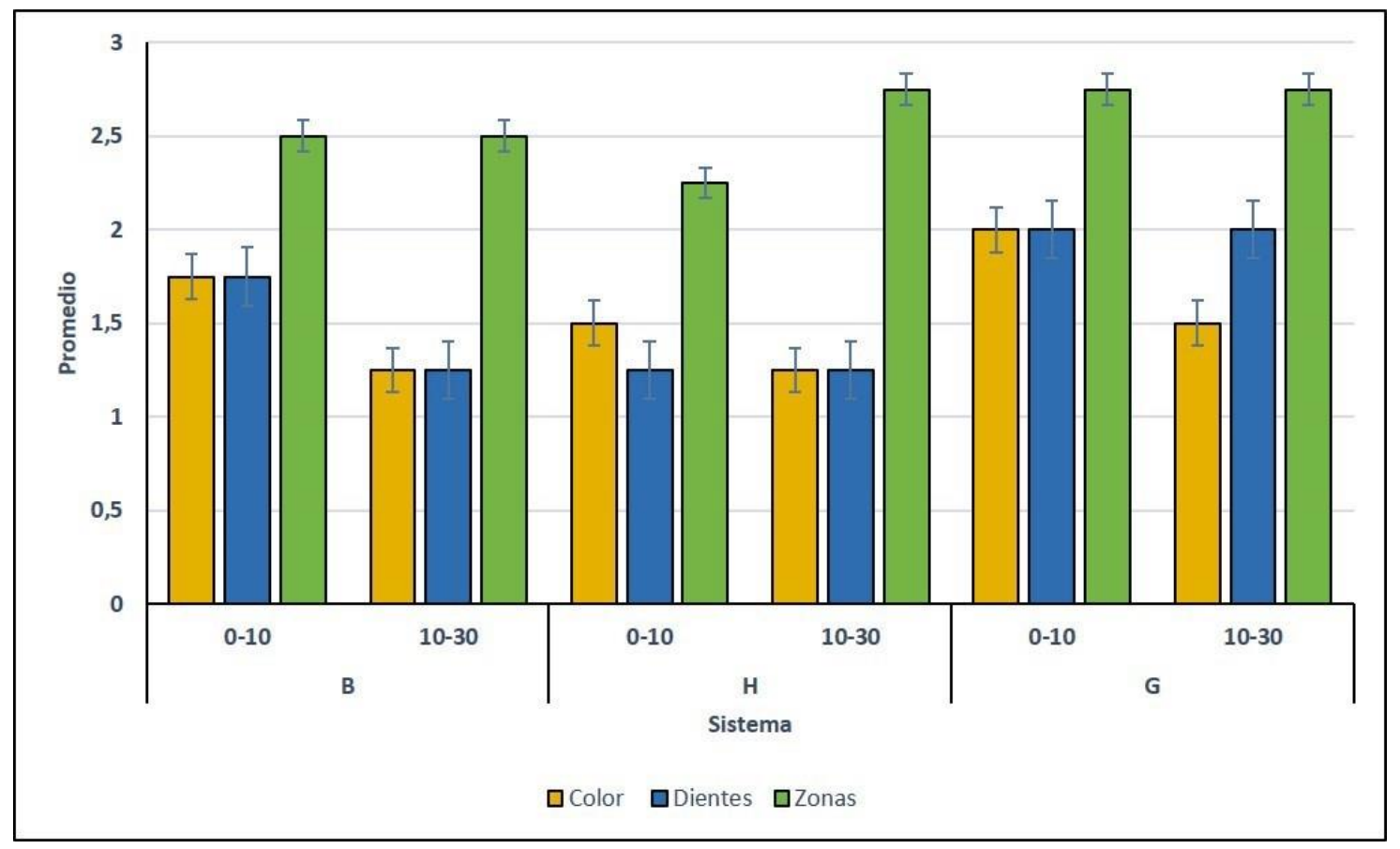

Fig. 5. Valores promedios de las características analizadas (color, dientes y zonas) por medio de CCP en los sistemas $\mathrm{B}, \mathrm{H}$ y G a profundidades de $0-10 \mathrm{~cm}$ y $10-30 \mathrm{~cm}$ para evaluar la calidad del suelo en una zona de bosque seco tropical.

Al confrontar los resultados obtenidos en laboratorio por técnicas analíticas estándar TAS con la CCP, se evidencia coherencia en los resultados obtenidos para cada parámetro evaluado, lo que indica que son técnicas complementarias para establecer características de calidad del suelo, que pueden ser empleadas para orientar su manejo.

Se observó que los CCP con menor número de dientes $(<D)$, colores oscuros $(<C)$ y desintegración entre zonas (Z), se encontraron en el sistema H. Además, reportaron tendencia a la compactación (>densidad). El sistema $B$, se encuentran asociado a suelos que presentan alteraciones en las relaciones catiónicas lo que ocasiona problemas de absorción de nutrientes por deficiencia inducida, mientras que los CCP reportaron $>C$ >D y >Z. En el sistema G, se evidencio contenido más alto de CO y zonas de los CCP menos disimiles; hay mejor integración lo que indica alta disponibilidad y posibilidad de acumulación y/o humificación de la materia orgánica en el suelo. Esto último coincide con lo reportado por Bezerra et al. (2019) quienes trabajando en sistemas agroforestales de una zona subtropical de Brasil, aplicaron la CCP y verificaron que las zonas con mayor contenido de CO mostraron mejoramiento en la calidad del suelo. 


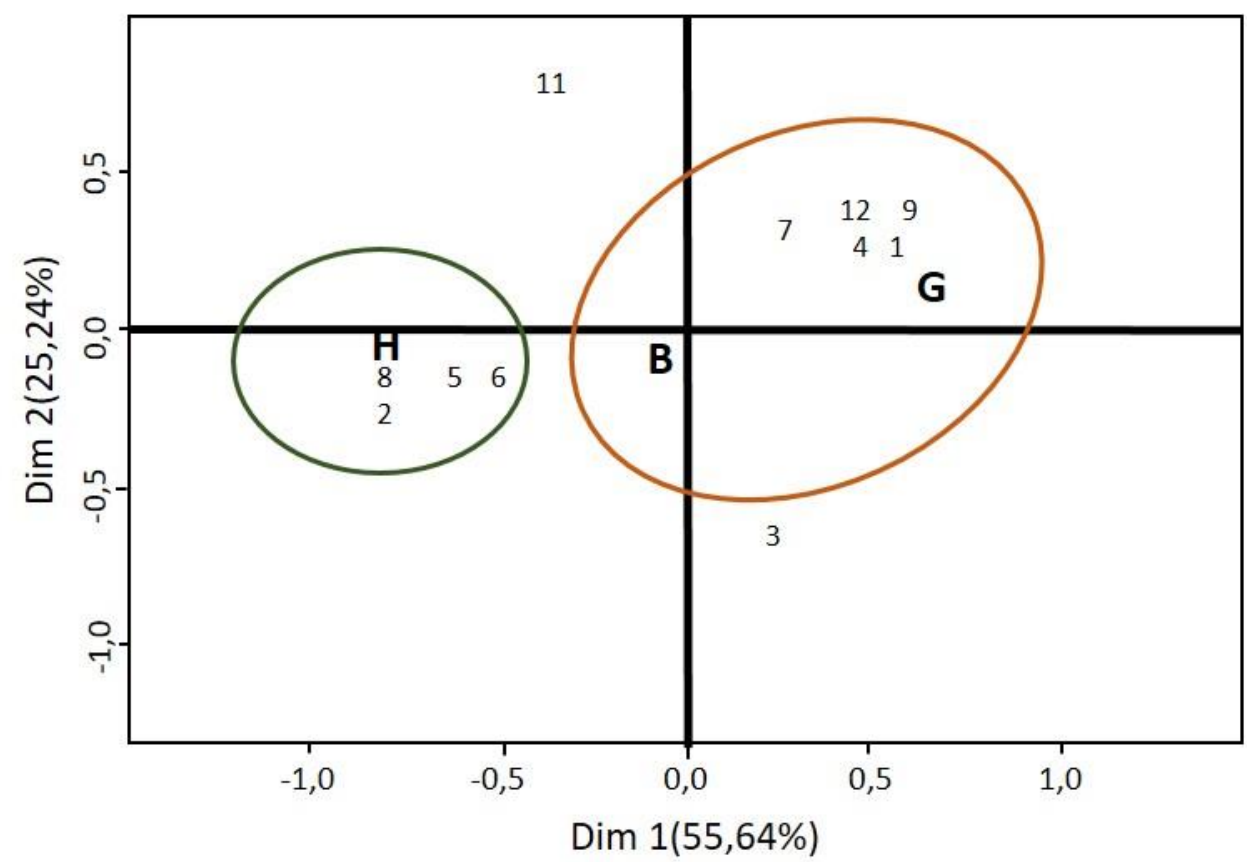

Fig. 6. Ordenamiento y determinación de las características evaluadas a partir de CCP en los sistemas de B, G y $\mathrm{H}$ en una zona de bosque seco tropical.

Burle y Figueiredo (2019) trabajando en una zona tropical de Sergipe, verificaron que la dilución de la solución extractora para analizar los contenidos de CO en sustratos a través de CCP, no es modificada al final del proceso lo cual es importante para realizar más estudios que verifiquen la calidad de los suelos. Un sistema de monitoreo confiable integra información específica y requiere indicadores del contexto que permitan monitorear en el tiempo, el comportamiento del recurso para definir medidas efectivas de desarrollo sostenible (Zambon et al., 2017, Salvati et al., 2015). Por lo que el conocimiento de técnicas con enfoque mixto cualitativo y cuantitativo para el monitoreo de parámetros de calidad del suelo como la CCP, en zonas vulnerables a desertificación, pueden ayudar a describir el camino evolutivo del territorio y orientar su manejo en condiciones donde las TAS son de alto costo y no se pueden realizar con frecuencia.

En Ecuador (trópico húmedo) Torres y Minga (2017) contrastaron el contenido de materia orgánica y la salud del suelo en sistemas de monocultivo de maíz y sistemas agroforestales. Se determinó contenido de materia orgánica en el laboratorio de la Universidad de Cuenca y a través de cromatografía se analizó la salud del recurso edáfico. Los resultados encontrados por estos investigadores generaron múltiples reflexiones desde el campo productivo-ecológico ratificando la cromatografía como herramienta de diagnóstico. En los suelos donde se desarrolló maíz convencional (monocultivo) por más de 15 años, se observó en los cromas colores oscuros hacia la zona central, con pérdida progresiva de interacción entre materia orgánica, humedad y microorganismos del suelo, se difumina el color hacia la periferia revelando, como se mostró en este trabajo, pérdida de interacción y disminución paulatina de microrganismos con procesos de pérdida estructural. Al igual que en nuestra investigación, los análisis de laboratorio ratificaron que los contenidos de materia orgánica son escasos. Los resultados de los cromas para suelos trabajados por productores agroecológicos, mostraron mejor interacción, se observó procesos de humificación, pero también algunas muestras evidencian materia orgánica sin descomponerse, posiblemente porque ocasionalmente algunos agricultores aplican agrotóxicos, la zona central es más oxigenada y activa, aunque en proporciones elevadas frente a su capacidad de humificación, la actividad microbiológica forma radiales que terminan en una buena coloración periférica. Pero existen algunas muestras que exhibieron proceso de compactación y pérdida de vida. En general, Torres y Minga (2017) afirman que el cultivo convencional mostró pérdida de biodiversidad y menor contenido de materia orgánica lo que concuerdan con lo obtenido en esta investigación, donde se evidenció diferencias entre los sistemas estudiados donde el cultivo convencional de hortalizas mostró falta de armonía en las características evaluadas.

De igual manera, Medina et al. (2018) efectuaron análisis de cromatogramas en diversos cultivos orgánicos del Cerro de Culiacán en México, observando coloraciones claras (doradas) indicativo de actividad mineral y contenido de materia orgánica, armonía e integración en las zonas de cromatogramas tal como se mostró para la zona $G$ y $B$ en esta investigación. En monocultivo, los cromatogramas presentaron coloraciones oscuras y terminaciones puntiagudas que revelan ausencia de materia orgánica y posiblemente agroquímicos. 
Esto pone en evidencia diferencias en técnicas de agricultura ancestral (orgánica) y la agricultura convencional. Tal como lo demuestran los resultados obtenidos en esta investigación, cuando se establecen diferencias entre los cromas mostrados por los suelos agrícolas $(H)$ frente a los encontrados en los bosques (B) y aquellos con cobertura permanente como son las gramas (G). Así mismo, cuando Medina y colaboradores evaluaron el bocashi (abono orgánico) en Santa Cruz de Ojo de Agua, mediante cromatografía, se evidenció buena evolución e integración de todas sus partes concluyendo que la CCP, es una herramienta para conocer las cualidades, condiciones y desarrollo que guardan las compostas y suelos en diversos cultivos y sistemas de producción que manifestaron características particulares propios de los sistemas de labranza. Los resultados obtenidos coinciden con los obtenidos por De Melo et al. (2019) en Brasil, donde se evaluó el uso de la CCP como técnica indicadora de la calidad de suelos en tres agro ecosistemas. Donde los resultados indicaron diferencias de calidad entre los suelos de los agroecosistemas, en índices deseables y contenido de materia orgánica. Sólo en el bosque se obtuvo una categoría excelente con buen contenido de MOS. se concluyó que la CP es una técnica que puede ser utilizada para evaluación cualitativa del suelo de ecosistemas naturales y agroecosistemas, siendo de simple manejo técnico y bajo costo, mostrándose como alternativa para la evaluación de calidad de suelos en sistemas agrícolas mixtos y productores pequeños que evalúen la salud integral del suelo.

Por otro lado, Chilon (2018) apreció microrganismos en compost con apoyo de la microscopía óptica, identificó hongos, actinomicetos y colonias de bacterias, a través de cromatografía circular. Los hallazgos obtenidos, comprobaron la necesidad de una determinación estricta de la calidad del compost, para evitar riesgos de contaminación de suelos agrícolas, donde la CCP da una caracterización general de la microbiota del suelo y el compost, que a su vez está relacionada con otros factores entre ellos actividad microbial y tipo de material orgánico, el autor destacó la homeostasis de algunos suelos tratados con compost, donde la microbiota actúa como parte de un sistema ordenado, llevando a cabo actividades específicas, secuenciales complementarias, sinérgicas y simbióticas, que permiten el mantenimiento y reproducción de la vida. Pero concluye que la técnica es general y hace falta interpretación detallada para analizar los cromas a profundidad. Si bien el trabajo aquí presentado no estudió fuentes como el compost, los resultados permiten verifica que los cromas con mejores distribuciones los muestran en aquellos suelos donde la intervención humana es mínima o donde se realizan acciones de mantenimiento o mejoramiento de suelos. Consecutivamente, Domigues et al. (2018) conciben que la agroecología es esencial en la sostenibilidad económica y ecológica de la agricultura, siendo uno de sus objetivos la conservación del suelo, promoviendo interacciones abióticas con los microorganismos para mantener su fertilidad. Esto implica afirmar que la cadena causal de factores que involucra procesos y características físicas, biológicas, químicas y energéticas, debe considerarse y que los métodos comunes de análisis del suelo no son suficientes para una evaluación completa, porque dependen de determinaciones cuantitativas y de parámetros individualmente analizados. Al igual a lo mostrado por esta investigación al estudiar los tres sistemas de uso de suelo, los métodos físicos, biológicos y químicos pueden llenar esta laguna dando una visión general de la interacción entre factores. Sin embargo, implica un análisis del suelo y su interpretación bajo enfoque multidimensional, que no siempre el más fácil. En este contexto la CCP es una herramienta auxiliar y alternativa de bajo costo que permite evaluar la fertilidad de los suelos en pequeñas parcelas.

Finalmente, se resalta que las características descritas en la CCP a pesar de no representar rangos cuantitativos específicos, ni describir minerales, reportaron resultados que presentan igual tendencia a los encontrados con técnicas analíticas estándar, lo cual amplia la visión holística del recurso. Igualmente, aunque este método ha sido en otros países en actividades de capacitación de pequeñas comunidades, las publicaciones científicas deben son insuficiente, para poder interpretar todos los resultados de manera puntual por lo que se requiere más investigación en el tema. Teniendo en cuenta la gran heterogeneidad de suelos y de agricultores sin acceso a tecnología de punta en Latinoamérica, esta técnica se convierte en una herramienta diagnostica en productores pequeños, los cuales han llamado la atención en el denominado desarrollo sostenible, tal así que el Plan de Acción Global 2019-2028 "Iniciativa regional de la Agricultura". Se expone como suelos sanos y en equilibrio sostienen la biodiversidad del planeta y son la base para la producción de alimentos saludables, constituye una herramienta que ayudan a combatir y adaptarse al cambio climático, almacenan agua, mejoran la resistencia a las sequías e inundaciones y son un recurso no renovable cuya conservación es esencial para la seguridad alimentaria y el futuro (FAO y IFAD, 2019). Por lo que es indispensable proteger y salvaguardar pequeños productores.

Al analizar los resultados obtenidos y compararlos con otros autores se evidencia que los suelos en mejor estado de salud son aquellos que han sido manejados con prácticas agrícolas sostenibles dirigidos por pequeños productores de minifundio o de agricultura mixta, (agrosistemas) o que están destinados a zonas 
de protección o sistemas intercalados, suelos que presentaron una cromatografía que refleja mayor integración entre sus zonas, colores claros con dientes redondeados, y que al ser comparados los resultados cualitativos con los métodos tradicionales coinciden con presencia de materia orgánica y mejores condiciones edáficas. Sin embargo, se reconoce la necesidad de profundizar en las interacciones de cada zona y correlacionar resultados y procedimientos con métodos cuantitativos que permitan una mejor interpretación de los cromas que plantean Burle y Figueiredo (2019), Chilon (2018), Domigues et al. (2018) y De Melo et al. (2019).

\section{CONCLUSIONES}

A partir de los resultados mostrados y de su análisis y de su discusión se pueden obtener las siguientes conclusiones: 1) El uso del suelo en el área de estudio determina la calidad y sostenibilidad del mismo, donde a medida que se reduce la cobertura vegetal, se evidenció deterioro en la calidad sistémica del recurso representada en incremento en los contenidos de sodio y de densidad aparente. 2) De los sistemas evaluados, las gramas mostraron el mejor comportamiento del recurso con mayores contenidos de carbono orgánico y zonas menos disímiles que denotan mayor calidad del recurso. 3) La cromatografía se constituye en herramienta de diagnóstico de suelos que puede complementarse con los métodos analíticos convencionales para obtener una visión holística de las características biológicas, químicas y físicas del recurso.

\section{AGRADECIMIENTOS}

Los autores agradecen el apoyo de la Universidad del Magdalena, a Fernando Manjarrez y a funcionarios del Laboratorio de Suelos de la Facultad de Ingeniería.

\section{REFERENCIAS}

Andrades, M. y M.E. Martínez, Fertilidad del Suelo y Parámetros que la Definen, 3a ed., 9-34. Universidad de La Rioja, Servicio de Publicaciones, La Rioja, España (2001).

Bautista, A., J. Etchevers, R. Del Castillo y C. Gutiérrez, La Calidad del Suelo y sus Indicadores, doi: 10.7818/re.2014.132.00; Rev. Ecosistemas, 13(2), 90-97 (2004).

Bertsch, F., Manual Para Interpretar la Fertilidad de Los Suelos de Costa Rica, 5-78. Universidad de Costa Rica, San José, Costa Rica (1987).

Bezerra, L., F. Silveira, V. Souza y R. Borsatto, Participatory construction in agroforestry systems in family farming: ways for the agroecological transition in Brazil, doi: 10.1080/21683565.2018.1509167; Agroecology and Sustainable Food Systems, 43(2), 180-200 (2019).

Briat, J.F., C. Dubos y F. Gaymard, Iron Nutrition, Biomass Production and Plant Product Quality, doi: 10.1016/j.tplants.2014.07.005; Trends in Plant Science, 20(1), 33-40 (2015).

Burle, E. y R. Figueiredo, Uso da Cromatografia Circular Plana em diferentes concentrações para análise de solo e de compostos orgánicos, Caderno de Graduação - Ciências Exatas e Tecnológicas - UNIT, 5(2), 19 (2019).

Costa, S., E.D. Souza y otros seis autores, Impact of an Integrated No-till Crop-Livestock System on Phosphorus Distribution, Availability and Stock, doi: 10.1016/j.agee.2013.12.001; Agriculture, Ecosystems y Environment, 190, 43-51 (2014).

Chilon C., Heurística del compost Altoandino, hallazgos científicos y su contribución al paradigma "Suelo Vivo", Apthapi, 4(2), 1212-1226 (2018).

De Melo, D., E. Dos Reis y otros tres autores, Cromatografia de pfeiffer como indicadora agroecológica da qualidade do solo em agroecossistemas, Revista Craibeiras de Agroecologia, 4(1), 7653-7658 (2019).

Domingues, S., R. Contini y otros cuatro autores, Conhecimento agroecológico a través da experimentação da cromatografia de pfeiffer, uma análise qualitativa dos solos, Revista da Jornada de Pós-Graduação e Pesquisa-Congrega Urcamp, 15(15) 932-940_(2018).

FAO y IFAD, Decenio de las naciones unidas para la agricultura familiar 2019-2028, Roma, Italia (2019).

FAO y GTIS, Estado Mundial del Recurso Suelo (en línea: http://www.fao.org/3/a-i5126s.pdf, acceso 5 de septiembre 2018), FAO, Roma, Italia (2016).

García, Y., W. Ramírez y S. Sánchez, Indicadores de la Calidad de los Suelos: Una Nueva Manera de Evaluar este Recurso; Pastos y Forrajes, 35(2), 125-38 (2012).

Gardner, W. K., High Sodium/Calcium Ratios in Alkaline Wimmera Soils: The Role of Magnesium and a Hypothesis for their Development, doi: 10.1080/01904167.2016.1225090; Journal of Plant Nutrition, 40(10), 1365-75 (2017).

Garratt, M., R. Bommarco y otros nueve autores, Enhancing Soil Organic Matter as a Route to the Ecological Intensification of European Arable Systems, doi: 10.1007/s10021-018-0228-2; Ecosystems, 21(7), 1404-15 (2018). 
González, J. y P. Ruiz, Investigación Cualitativa Versus Cuantitativa: ¿Dicotomía Metodológica o Ideológica?, doi.org/10.4321/S1132-12962011000200011; Index de Enfermería, 20(3), 189-93 (2011).

IDEAM y U.D.C.A., Síntesis Estudio Nacional de la Degradación de Suelos por Erosión en Colombia (en línea: https://goo.gl/K9MkaD, acceso enero 27 2018), IDEAM - UDCA, Bogotá (2015).

Jia, Z., Y. Kuzyakov, D. Myrold y J. Tiedje, Soil Organic Carbon in a Changing World, doi.org/10.1016/S10020160(17)60489-2; Pedosphere, Special Issue on Soil Organic Carbon in a Changing World, 27(5), 789-91 (2017)

Jin, K., P. White y otros tres autores, Shaping an Optimal Soil by Root-Soil Interaction, doi.org/10.1016/j.tplants.2017.07.008; Trends in Plant Science, 22(10), 823-29 (2017).

Keesstra, S. D., J. Bouma, y otros 13 autores, The Significance of Soils and Soil Science towards Realization of the United Nations Sustainable Development Goals, doi.org/10.5194/soil-2-111-2016; Soil, 2(2), 111-28 (2016).

Kokornaczyk, M., F. Primavera y otros tres autores, Analysis of Soils by Means of Pfeiffer's Circular Chromatography Test and Comparison to Chemical Analysis Results, doi.org/10.1080/01448765.2016.1214889; Biological Agriculture \& Horticulture, 33(3), 143-57 (2017).

Medina, T., G. Arroyo y otros cuatro autores, Cromatografía de Pfaiffer en el Análisis de Suelos de Sistemas Productivos, doi.org/10.29312/remexca.v9i3.1223, Revista Mexicana de Ciencias Agrícolas, 9(3), 665-73 (2018).

Mengel, K. y E. A. Kirkby, Principles of Plant Nutrition. 5.a ed., 15-663, Springer, Netherlands (2006).

Molina, E., Análisis de Suelos y su Interpretación (en línea: https://goo.gl/G4ngmP, acceso marzo 20 2018), Aminogrow Internacional, San José de Costa Rica (2007).

Nagargade, M., V. Tyagi y M. Kumar, Climate Smart Agriculture: An Option for Changing Climatic Situation; in Plant Engineering by Snježana Jurić, IntechOpen pp. 143-165 (2017).

Nikolaidis, N., Human Impacts on Soils: Tipping Points and Knowledge Gaps, doi.org/10.1016/j.apgeochem.2011.03.111; Applied Geochemistry, 26: S230-S233 (2011).

Pfeiffer, E., Chromatography Applied to Quality Testing, 5-47, Bio-Dynamic Farming and Gardening Assoc., Stroudsburg, Pensilvania, Estados Unidos (1984).

Rangel, J. y J. Carvajal, Clima de la Región Caribe Colombiana. En Colombia Diversidad Biótica XII: La Región Caribe de Colombia, Rangel, J. 67-129. Bogotá, Colombia: Instituto de Ciencia Naturales, Universidad Nacional de Colombia (2012).

Restrepo, J. y S. Pineiro, Cromatografía, Imágenes de Vida y Destrucción del Suelo, 21a ed., 29-240. Feriva, Cali, Colombia (2011).

Rossel, R., E. Viscarra y otros tres autores, Mapping Iron Oxides and the Color of Australian Soil Using Visible-Nearinfrared Reflectance Spectra, doi.org/10.1029/2009JF001645; Journal of Geophysical Research: Earth Surface, 115 (F4), 1-13 (2010).

Salvati, L., C. Ferrara y P. Corona, Indirect validation of the Environmental Sensitive Area Index using soil degradation indicators: A country-scale approach. Ecological indicators, 57, 360-365 (2015).

Sas, L., H. Marschner, V. Römheld y S. Mercik, Effect of Nitrogen Forms on Growth and Chemical Changes in the Rhizosphere of Strawberry Plants, doi.org/10.1007/s11738-003-0004-5; Acta Physiologiae Plantarum, 25(3), 241-47 (2003)

Smith, P., J. House y otros 19 autores, Global Change Pressures on Soils from Land Use and Management, doi.org/10.1111/gcb.13068; Global Change Biology, 22(3), 1008-28 (2016).

Torres, M. E. y N. Minga, La Cuestión Tecnológica En La Matriz Productiva Del Maíz-Agronegocio y Agroecología en Pindal y Calvas Loja Ecuador, Revista Académica-Investigativa del Área Jurídica Social y Administrativa, 6(1), 49-58 (2019).

Vásquez, J., D. Baena y J. Menjivar, Variabilidad Espacial de Propiedades Físicas y Químicas en Suelos de la Granja Experimental de la Universidad del Magdalena (Santa Marta, Colombia); Acta Agronómica, 59(4), 449-456 (2010).

Vásquez, R. y F. Macías, Fraccionamiento Químico del Carbono en Suelos con Diferentes Usos en el Departamento de Magdalena, Colombia; Terra Latinoam., 35(1), 7-17 (2017).

Walkley, A. y I. Black, An Examination of the Degtjareff Method for Determining Soil Organic Matter, and a proposed modification of the chromic acid titration method; Soil Science, 37(1), 29-38 (1934).

Wolf, B. y G. Snyder, Sustainable Soils: The Place of Organic Matter in Sustaining Soils and Their Productivity, 19-182, CRC Press. New York, Estados Unidos (2003).

Zambon, I., A. Colantoni y otros cuatro autores, Land Quality, Sustainable Development and Environmental Degradation in Agricultural Districts: A Computational Approach Based on Entropy Indexes, doi.org/10.1016/j.eiar.2017.01.003; Environmental Impact Assessment Review, 64, 37-46 (2017). 
